\title{
Sélection de résumés - Congrès 2010 des étudiants de la Société canadienne d'épidémiologie et de biostatistique (SCEB)
}

C'est en mai 2010, lors du congrès des étudiants de la Société canadienne d'épidémiologie et de biostatistique (SCEB), que les responsables de la revue Maladies chroniques au Canada (MCC) ont annoncé le concours de résumés à l'intention des étudiants. Chaque texte soumis dans le cadre de ce concours allait faire l'objet d'un examen par un comité de l'Agence de la santé publique. L'objectif était de choisir les 10 meilleurs textes en matière de maladies chroniques afin de les publier dans le numéro du mois de mars 2011.

Les étudiants se sont montrés très intéressés par ce concours. En effet, nous avons reçu 57 résumés sur les maladies chroniques et les traumatismes. La sélection a été faite par un jury composé de :

- Howard Morrison, Ph. D., rédacteur en chef de Maladies chroniques au Canada, Agence de la santé publique du Canada;

- Kenneth Johnson, Ph. D., épidémiologiste principal, Agence de la santé publique du Canada;

- Fabiola Tatone-Tokuda, M. Sc., comité de rédaction de Maladies chroniques au Canada et Université d'Ottawa;

- Michelle Tracy, M.A., gestionnaire de la rédaction de Maladies chroniques au Canada, Agence de la santé publique du Canada.
Les membres du comité ont évalué les résumés en fonction des critères suivants : originalité, clarté, excellence scientifique et technique, impact potentiel.

Depuis 2009, MCC collabore avec la SCEB afin de faciliter l'accès des étudiants à la publication. À la suite du congrès des étudiants de la SCEB en mai 2009, MCC a accepté de publier tous les résumés retenus dans le cadre du congrès conjointement avec le Relevé des maladies transmissibles au Canada. Vous pouvez consulter les résumés relatifs aux maladies chroniques à http://www.phac-aspc.gc.ca/publicat/ cdic-mcc/30-2/cseb_01-fra.php et ceux traitant des maladies infectieuses à http:// www.phac-aspc.gc.ca/publicat/cdic-mcc/ abstract/index-fra.php.

La revue MCC est très heureuse de soutenir les étudiants dans leurs projets de publication. Toutes nos félicitations aux gagnants!

Michelle Tracy, M.A.

Gestionnaire de la rédaction, Maladies chroniques au Canada 


\title{
Utilisation de l'indice de longueur du membre inférieur chez l'enfant comme facteur prédictif de l'embonpoint et de l'obésité à l'adolescence
}

\begin{abstract}
Akseer N. (1); Liu J. (2); Hay J. (3); Faught B. (4); Wade T. (4); Cairney J. (4)
Contexte/But/Objectifs : La hausse de la prévalence de l'embonpoint et de l'obésité dans la population pédiatrique est devenue une préoccupation croissante en santé publique dans de nombreux pays. Notre étude visait à déterminer si les composantes de l'évaluation de la taille des enfants, en particulier l'indice de longueur du membre inférieur (ILMI $=$ [taille debout - taille assise]/ taille), peuvent servir à évaluer le risque d'embonpoint et d'obésité à l'adolescence.

Plan d'étude/Méthodologie : Au début de l'étude, on a mesuré la taille debout et assise d'environ 2360 élèves de la région

de Niagara. Cinq ans plus tard, on a pesé et mesuré 1167 d'entre eux (573 filles et 594 garçons). On a établi le seuil de l'embonpoint et de l'obésité à un indice de masse corporel (IMC, en $\mathrm{kg} / \mathrm{m}^{2}$ ) adapté à l'âge et au sexe et équivalent à un IMC supérieur à 25 chez l'adulte.

Résultats : Globalement, $34 \%$ ( $\mathrm{n}=298)$ des adolescents ont été considérés comme souffrant d'embonpoint ou obèses. Les résultats de l'analyse de régression logistique ont indiqué que, pour une augmentation de l'ILMI de 1 unité, le risque de faire de l'embonpoint ou d'être obèse diminuait

de $24 \%(\mathrm{RC}=0,76$; IC à $95 \%: 0,66$ à 0,87 ) après ajustement selon l'âge, le sexe et la circonférence de la taille au début de l'étude. Les autres ajustements effectués pour tenir compte des variables confusionnelles liées au jeune âge n'ont pas modifié cette relation.

Conclusions : L'ILMI mesuré pendant l'enfance peut servir à prédire le risque d'embonpoint et d'obésité à l'adolescence. Toutefois, le mécanisme sous-jacent n'est pas clair et d'autres études sont nécessaires. L'IMC et le tabagisme de la mère peuvent influencer l'ILMI chez l'enfant.
\end{abstract}

\section{Caractéristiques associées aux besoins insatisfaits en matière de soutien chez les familles avec enfant d'âge scolaire atteint d'un trouble du spectre autistique}

\author{
Brown H.K. (5); Ouellette-Kuntz H. (5); Hunter D. (5); Kelley E. (6)
}

Contexte/But/Objectifs : Étant données les fortes pressions exercées sur les services destinés aux personnes atteintes d'autisme, cette étude visait à 1) décrire les besoins insatisfaits en matière de soutien signalés par les parents d'enfants d'âge scolaire atteints d'un trouble du spectre autistique et à 2) analyser ces besoins en fonction de l'autonomie fonctionnelle de l'enfant. Nous avons posé l'hypothèse que les familles avec enfant ayant une autonomie fonctionnelle faible ou élevée auraient des besoins de soutien moins bien satisfaits que les familles avec enfant ayant une autonomie fonctionnelle modérée.
Plan d'étude/Méthodologie : Nous avons réalisé une enquête transversale portant sur 77 familles avec enfant ayant un trouble du spectre autistique à l'aide du Family Needs Questionnaire (questionnaire sur les besoins des familles) et de la version révisée des échelles de comportements indépendants. L'autonomie fonctionnelle des enfants était définie par leur capacité d'adaptation et leurs troubles de comportement. L'âge moyen des enfants était de 9,6 ans (ÉT $=2,1$ ) et 85,7 \% d'entre eux étaient des garçons.

Résultats : Une modélisation linéaire généralisée a montré, après ajustement pour tenir compte des autres variables, que les différences entre les besoins insatisfaits chez les enfants à autonomie fonctionnelle faible et chez les enfants à autonomie fonctionnelle modérée n'étaient pas significatives (RT $=1,27$; IC à $95 \%: 0,67$ à $2,38)$. Contrairement à notre hypothèse, les enfants qui avaient une autonomie fonctionnelle élevée avaient un risque significativement plus faible de présenter des besoins insatisfaits que les enfants ayant une autonomie fonctionnelle modérée $(\mathrm{RT}=0,61$; IC à $95 \%: 0,43$ à 0,85$)$.

Conclusions : Des données sur les besoins insatisfaits peuvent aider les décideurs et les fournisseurs de services à distribuer efficacement les ressources limitées.

\footnotetext{
Rattachement

1. Faculté des sciences de la santé appliquées, Université Brock, St. Catharines (Ontario), Canada

2. Département des sciences de la santé communautaire, Université Brock, St. Catharines (Ontario), Canada

3. Département de psychiatrie et de neurosciences comportementales, Université McMaster, Hamilton (Ontario), Canada

4. Département de médecine familiale, Université McMaster, Hamilton (Ontario), Canada

5. Département de santé communautaire et d'épidémiologie, Université Queen's, Kingston (Ontario), Canada

6. Département de psychologie, Université Queen's, Kingston (Ontario), Canada
} 


\section{Temps passé devant un écran et comportements à risque chez les jeunes Canadiens de 10 à 16 ans}

Carson V. (1); Pickett W. (2,3); Janssen I. (1,2)

Contexte/But/Objectifs : Cette étude vise à examiner l'utilisation de la télévision, de l'ordinateur et des jeux vidéo en tant que déterminant possible de comportements à risque multiples (CRM) chez les jeunes Canadiens.

Plan d'étude/Méthodologie : Les résultats étaient basés sur l'enquête Les comportements de santé des jeunes d'âge scolaire menée au Canada en 2005-2006, qui portait sur un échantillon transversal représentatif de 8215 jeunes de la $6^{\mathrm{e}}$ à la $10^{\mathrm{e}}$ années et un échantillon longitudinal d'une année regroupant 1424 jeunes de la $9^{\mathrm{e}}$ et de la $10^{\mathrm{e}}$ années. Les participants ont été classés en quartiles en fonction du nombre total d'heures par semaine passées devant la télévision, à des jeux vidéo et à l'ordinateur. Six variables représentant des comportements à risque (tabagisme, ébriété, non-port de la ceinture de sécurité, consommation de cannabis, consommation de drogues illicites, non-utilisation du condom) ont été combinées pour obtenir le score de CRM. Nous avons eu recours à des modèles de régression logistique à mesure ordinale et répétée pour examiner les associations entre le temps passé devant un écran et les variables de CRM.

Résultats : Une utilisation importante de l'ordinateur est associée à une augmentation des CRM d'environ 50 \% dans les deux échantillons. De longues heures passées devant la télévision sont également associées à une modeste hausse des CRM dans l'échantillon transversal.

Conclusions : L'usage important de l'ordinateur est l'activité à l'écran la plus fortement associée aux CMR. Les travaux futurs devraient se pencher sur la relation dose-réponse entre certaines activités devant l'écran et la santé des adolescents, de manière à renforcer les lignes directrices actuellement destinées aux jeunes et portant sur le temps passé devant un écran.

\section{Prévalence et facteurs de risque de l'asthme chez les enfants autochtones vivant hors réserve, au Canada}

Chang H.J. (4); Senthilselvan A. (4)

Contexte/But/Objectifs : L'asthme est une affection chronique courante chez les enfants; toutefois, seules quelques études ont étudié la morbidité liée à l'asthme chez les enfants autochtones canadiens. Cette étude vise à déterminer chez ces derniers la prévalence de l'asthme et les facteurs de risque d'avoir déjà souffert d'asthme (" asthme passé ») ou de souffrir actuellement d'asthme (« asthme actuel»).

Plan d'étude/Méthodologie : Nous avons utilisé les données sur les enfants de 6 à 14 ans de l'Enquête auprès des peuples autochtones de 2006. Plus de 15000 enfants vivant hors réserve ont participé à cette enquête. Un enfant était considéré comme asthmatique si un parent faisait état d'un diagnostic d'asthme posé par un professionnel de la santé.

Résultats : Chez les enfants autochtones canadiens vivant hors réserve, la prévalence de l'asthme passé était de 14,3\%, celui de l'asthme actuel de 5,7 \%. La prévalence de l'asthme était significativement plus faible chez les enfants d'origine inuite que chez les enfants des Premières nations ou métis. Les facteurs de risque significatifs de l'asthme passé comprenaient le fait d'être de sexe masculin, les allergies, un faible poids à la naissance, l'obésité, un logement nécessitant des réparations et le fait d'habiter en milieu urbain. Les associations entre l'asthme passé et un faible revenu familial, la fréquentation d'une garderie et des troubles psychologiques étaient presque significatives. Les facteurs de risque de l'asthme actuel étaient semblables à ceux de l'asthme passé.

Conclusions : Dans notre étude, les facteurs de risque d'avoir déjà souffert d'asthme chez les enfants autochtones vivant hors réserve étaient semblables à ceux signalés chez les enfants non autochtones. 


\title{
Demandes de radiographies en cas de blessures aiguës à la cheville à Kingston (Ontario) : une évaluation du respect des règles d'Ottawa par les médecins
}

\author{
Dowdall H. (1); Nichol M. (1); Brison R. (1,2); Pickett W. (1,2)
}

\begin{abstract}
Contexte/But/Objectifs : Les règles d'Ottawa concernant les blessures à la cheville (Ottawa Ankle Rules) ont été formulées en 1992; il s'agit de règles décisionnelles cliniques fondées sur des données probantes permettant de déterminer la nécessité de prendre des radiographies au cours d'une consultation au service d'urgence. Nous avons évalué le respect de ces règles au fil du temps dans deux services d'urgence hospitaliers de Kingston, à l'aide d'une étude par couplage d'enregistrements.
\end{abstract}

Plan d'étude/Méthodologie : Dans cette étude de population rétrospective, nous avons associé les cas de blessures à la cheville survenues à Kingston consignées dans le Système canadien hospitalier d'information et de recherche en prévention des traumatismes (2001-2007) aux dossiers de congé d'hôpital contenant les codes d'interventions. Nous avons analysé l'évolution de la proportion de blessures à la cheville ayant fait l'objet d'une demande de radiographie et nous avons comparé ces proportions au taux de référence de 1993, soit l'année ayant suivi l'implantation des règles d'Ottawa.

Résultats : Comparativement au taux de référence initial de 57,6 \% (IC à $95 \%$ : 55,3 à 59,9 ) noté à la suite de l'implantation des règles d'Ottawa, l'analyse de 7706 blessures aiguës à la cheville a montré que la proportion de blessures à la cheville faisant l'objet d'une demande de radiographie avait augmenté (p. ex., en 2007, 70,3 \% [IC à $95 \%: 67,3$ à 72,9$])$. La proportion de cas diagnostiqués comme étant des fractures reste similaire au cours de la période $(18,3 \%$ à $21,9 \%)$.

Conclusions : Les médecins doivent respecter les règles d'Ottawa pour diminuer les coûts au sein des services d'urgence et pour réduire l'exposition des patients aux radiographies.

\section{Relation entre l'adiposité et le score de Gleason chez les hommes atteints de cancer de la prostate localisé}

Hack E.E. (3); Siemens D.R. (4); Groome P.A. (3)

Contexte/But/Objectifs : L'association entre l'adiposité et l'agressivité du cancer de la prostate $(\mathrm{CaP})$ est controversée. Nous avons examiné la relation entre l'indice de masse corporel (IMC) au moment du diagnostic de CaP et le phénotype malin mesuré par le score de Gleason.

Plan d'étude/Méthodologie : Dans cette étude transversale menée en Ontario chez 1096 patients atteints de CaP recevant un traitement curatif, les données d'une base électronique ont été complétées par un examen rétrospectif des dossiers médicaux. L'IMC a été classé en trois catégories : poids normal $\left(\leq 25,0 \mathrm{~kg} / \mathrm{m}^{2}\right)$, embonpoint $\left(25,0\right.$ à $\left.29,9 \mathrm{~kg} / \mathrm{m}^{2}\right)$ et obésité $\left(\geq 30 \mathrm{~kg} / \mathrm{m}^{2}\right)$. Nous avons aussi étudié l'influence de la présence ou non de diabète. Nous avons classé les scores de Gleason en catégories ayant une signification clinique : 2 à 4,5 à 6,7 et 8 à 10 .

Résultats : L'IMC n'était pas associé au score de Gleason : 9,7 \% des sujets ayant un IMC normal avaient un score de Gleason supérieur ou égal à 8; inversement, 9,4% des sujets classés dans cette catégorie de score de Gleason présentaient un embonpoint ou étaient obèses $(p=0,73)$. Parmi les sujets diabétiques, $11,7 \%$ avaient un score de Gleason supérieur ou égal à 8 , comparativement à 9,3\% des sujets non diabétiques ( $p=0,79)$. Après stratification selon l'âge, la distribution du score de Gleason dans la plus jeune cohorte de sujets obèses se rapprochait des scores de Gleason les plus élevés ( $p=0,13)$.

Conclusions : Ni un IMC élevé, ni le fait d'être atteint de diabète au moment du diagnostic ne semblaient être associés au score de Gleason. L'influence de l'adiposité sur le CaP devrait faire l'objet d'autres études, une attention particulière devant être portée à la durée de l'exposition à un IMC élevé.

\footnotetext{
Rattachement

1. Département de santé communautaire et d'épidémiologie, Université Queen's, Kingston (Ontario), Canada

2. Département de médecine d'urgence, Université Queen's, Kingston (Ontario), Canada

3. Division des soins contre le cancer et d'épidémiologie, Institut de recherche sur le cancer de l'Université Queen's, Kingston (Ontario), Canada

4. Département d'urologie, Université Queen's, Kingston (Ontario), Canada
} 


\title{
Départs contre avis médical après un traumatisme cérébral : une blessure intentionnelle est-elle une variable explicative?
}

\author{
Kim H. (1); Colantonio A. (2); Bayley M. (2); Dawson D. (3)
}

Contexte/But/Objectifs : Les départs contre avis médical (DCAM) sont régulièrement signalés comme étant à l'origine de moins bons résultats sur le plan fonctionnel et de fréquentes réadmissions à l'hôpital. Cette étude vise à élaborer le profil de risque des patients partis contre avis médical et atteints de traumatisme cérébral (TC), ainsi qu'à examiner les facteurs associés aux DCAM et à déterminer si les blessures intentionnelles sont une variable explicative des DCAM.

Plan d'étude/Méthodologie : Nous avons effectué une étude de cohorte rétrospective à l'aide des données sur les congés d'hôpital d'un ensemble de données minimales (EDM) du Registre ontarien des traumatismes (ROT) pour les années 1993-1994 et 2000-2001. Nous avons relevé 15684 cas de TC; 446 (2,84 \%) d'entre eux étaient associés à une DCAM.

Résultats : Le nombre de DCAM était significativement plus élevé chez les sujets dont le TC était intentionnel que chez les sujets où il ne l'était pas. Les patients dont les blessures étaient intentionnelles avaient quitté l'hôpital contre avis médical deux fois plus souvent que ceux dont les blessures n'étaient pas intentionnelles, après ajustement pour les autres facteurs. Les plus jeunes dans la tranche des 25 à 54 ans et ceux ayant des antécédents d'abus d'alcool et de drogues étaient davantage susceptibles de partir contre avis médical.

Conclusions : Les patients atteints de TC quittant l'hôpital contre avis médical forment une population à risque. Les blessures intentionnelles sont un facteur indépendant important à prendre en compte lorsqu'on effectue des études de modélisation des DCAM. Le repérage précoce des patients dont le TC est intentionnel contribuerait à l'amélioration des stratégies de prévention, et donc de la prestation des soins de santé et des résultats en matière de santé.

\section{Réadaptation après un traumatisme cérébral intentionnel : changements fonctionnels et destinations après le congé d'hôpital}

Kim H. (1); Colantonio A. (2); Bayley M. (2); Dawson D. (3)

Contexte/But/Objectifs : Les traumatismes cérébraux (TC) d'origine violente sont un enjeu de santé publique majeur. En effet, selon un récent rapport de l'Institut canadien d'information sur la santé (ICIS), le nombre de TC intentionnels augmente chez les jeunes adultes. Cette étude vise à comparer les changements fonctionnels et les destinations après le congé d'hôpital des victimes de TC intentionnels et des victimes de TC non intentionnels.

Plan d'étude/Méthodologie : Pour réaliser cette étude de cohorte prospective, nous avons utilisé les enregistrements sur les soins actifs et la réadaptation des patients hospitalisés à l'échelle de la population, la Base de données sur les congés des patients (BDCP) et le Système national d'information sur la réadaptation (SNIR) de l'ICIS. Pour mesurer les résultats en matière de réadaptation, nous avons analysé les améliorations fonctionnelles absolues et relatives à l'aide de sous-échelles motrices et cognitives, ainsi que les destinations après le congé d'hôpital.

Résultats : Les victimes de TC intentionnels présentaient des améliorations absolues des fonctions motrices et des améliorations relatives des fonctions cognitives significativement plus faibles dans les analyses unidimensionnelles au moment du congé d'hôpital. Les TC intentionnels étaient également associés à de plus faibles améliorations relatives des fonctions cognitives, après ajustement pour l'âge, le sexe, les antécédents d'abus d'alcool ou de drogues et d'autres variables cliniques et démographiques. Les sujets atteints de TC intentionnels étaient moins fréquemment renvoyés à leur domicile après leur congé d'hôpital (après ajustement pour les autres variables indépendantes).

Conclusions : Les victimes de TC intentionnels forment une population distincte dans le milieu de la réadaptation au Canada. Au cours de leur réadaptation à l'hôpital, il faudrait insister sur les améliorations réelles des fonctions cognitives. Étant donné leur destination différente au départ de l’hôpital, il faudrait également procéder à une planification plus attentive de leur congé d'hôpital.

\footnotetext{
Rattachement

1. Secteur des sciences de la réadaptation, Université de Toronto, Toronto (Ontario), Canada

2. Institut de réadaptation de Toronto, Université de Toronto, Toronto (Ontario), Canada
}

3. Unité de recherche appliquée Kunin-Lunenfeld, Baycrest, Toronto (Ontario), Canada 


\title{
Soutien social en général et au travail, et risque de récidive après un premier infarctus du myocarde : étude prospective sur 6 ans
}

\author{
Thiam A. (1); Brisson C. (1)
}

Contexte/But/Objectifs : Cette étude a examiné l'effet d'un faible soutien social en général (SSG) et d'un faible soutien social au travail (SST) sur le risque de coronaropathie récurrente.

Plan d'étude/Méthodologie : Une cohorte de 972 femmes et hommes ayant subi un premier infarctus du myocarde aigu a été recrutée dans les hôpitaux du Québec. Au cours du suivi de 6 ans, trois entrevues ont été réalisées, et nous avons examiné les dossiers médicaux en vue de repérer la survenue des coronaropathies suivantes : coronaropathie fatale, infarctus du myocarde non fatal et angine instable. Nous avons évalué le SSG (soutien émotionnel ou informationnel et interactions sociales positives) à l'aide de la version française de l'échelle MOS (Medical Outcomes Study Social Support Survey) et le SST (relations avec les collègues et le superviseur) à l'aide du questionnaire de Faucett, basé sur l'Interpersonal Relationships Inventory (inventaire des relations interpersonnelles). Nous avons utilisé la régression de Cox pour l'analyse de données.

Résultats : En tout, 206 incidents liés à la coronaropathie sont survenus lors du suivi. On a observé une association modérée significative entre l'exposition continue à un bas SSG et le risque de coronaropathie récurrente (rapport de risques [hazard ratio] ajusté $=1,66$; IC : 1,08 à 2,57$)$. Aucune association n’a été constatée entre un bas SST et le risque de coronaropathie récurrente.

Conclusions : Notre étude indique que les patients ayant subi un infarctus du myocarde qui ont peu de soutien social en général (mais pas spécifiquement au travail) courrent un risque accru d'être atteints d'une coronaropathie récurrente. Nous avons illustré également qu'il est nécessaire d'effectuer d'autres études sur ce thème.

\section{Logements inadéquats et facteurs de risque associés chez les personnes transsexuelles en Ontario : une étude transversale}

Warner A. J. (2); Bauer G. R. (2); Scanlon K. (3); Pyne J. (4)

Contexte/But/Objectifs : Les conditions de logement inadéquates et la discrimination en matière de logement, présentes au Canada comme à l'échelle mondiale, nuisent à la santé et au bien-être des personnes touchées. Un taux disproportionnellement élevé de personnes transsexuelles (trans) sont sans abri, ce qui peut compromettre leur capacité à avoir accès à un logement sûr et stable. Dans le cadre de cette étude, nous avons estimé la fréquence des logements inadéquats, évalué les facteurs de risque potentiels et conçu un modèle exploratoire de prévision des facteurs de risque définis pour la population trans.
Plan d'étude/Méthodologie : Les données sont tirées de l'enquête Trans PULSE, une enquête transversale communautaire sur les personnes trans demeurant en Ontario ( $n=433$ ). Nous avons effectué des analyses unidimensionnelles et bidimensionnelles à l'aide d'un modèle prédictif logistique des conditions de logement inadéquates, pondérées en fonction de la probabilité de recrutement.

Résultats : On a estimé que 33,1\% des personnes trans en Ontario vivent dans des conditions de logement inadéquates. Les facteurs de risque significatifs dans le modèle prédictif à plusieurs variables comprennent un revenu personnel annuel inférieur à
$15000 \$(\mathrm{RC}=3,57$; IC à $95 \%: 1,18$ à 11,11 ; référence : revenu annuel supérieur à 30000 \$), le fait d'être toujours perçu par les autres comme une personne trans ( $\mathrm{RC}=$ 7,14 ; IC à $95 \%: 1,08$ à 50,00; référence : jamais) et la consommation très fréquente d'alcool $(\mathrm{RC}=2,72$; IC à $95 \%: 1,03$ à 7,19; référence : ne jamais consommer d'alcool).

Conclusions : Ces données suggèrent l'existence d'une discrimination en matière de logement, qui rendrait les personnes trans plus vulnérables à des conditions de logement inadéquates. D’autres études sont nécessaires pour continuer d'améliorer et réévaluer les services et les politiques destinés aux communautés trans.

\footnotetext{
Rattachement

1. URESP, Centre de recherche FRSQ du Centre hospitalier affilié universitaire de Québec, Québec (Québec), Canada

2. Département d'épidémiologie et de biostatistique, École de médecine et de dentisterie Schulich, Université de Western Ontario, London (Ontario), Canada

3. The 519 Church Street Community Centre, Toronto (Ontario), Canada

4. Projet Trans PULSE (Ontario), Canada
} 